\section{Cahiers de Narratologie}

Analyse et théorie narratives

13 | 2006

Nouvelles approches de l'intertextualité

\title{
Intertextualité et symbolisation : la poétique d'Arthur Symons
}

\section{Micéala Symington et Bernard Franco}

\section{(2) OpenEdition}

\section{Journals}

Édition électronique

URL : http://journals.openedition.org/narratologie/352

DOI : 10.4000/narratologie.352

ISSN : 1765-307X

Éditeur

LIRCES

\section{Référence électronique}

Micéala Symington et Bernard Franco, «Intertextualité et symbolisation : la poétique d'Arthur Symons », Cahiers de Narratologie [En ligne], 13 | 2006, mis en ligne le 01 septembre 2006, consulté le 15 novembre 2019. URL : http://journals.openedition.org/narratologie/352 ; DOI : 10.4000/ narratologie.352

\section{Ce document a été généré automatiquement le 15 novembre 2019.}

\section{c) $(1) \odot$}

Cahiers de Narratologie - Analyse et théorie narratives est mis à disposition selon les termes de la licence Creative Commons Attribution - Pas d'Utilisation Commerciale - Pas de Modification 4.0 International. 


\title{
Intertextualité et symbolisation : la poétique d'Arthur Symons
}

\author{
Micéala Symington et Bernard Franco
}

1 C'est peut-être Mallarmé qui offre la formulation la plus extrême des implications poétiques de l'intertextualité lorsqu'il affirme «que, plus ou moins, tous les livres contiennent la fusion de quelques redites comptées $»^{1}$. Les « redites comptées », par la double référence à l'oralité de la légende, insistent ici sur une mise en fiction déjà acquise du référent poétique. Autrement dit, c'est la connexion du texte avec un univers culturel qui lui permet de conduire à « la notion pure $»^{2}$ qui définit l'idéal de toute poésie.

2 Gérard Genette, dans sa propre définition de l'intertextualité, semble formaliser cet idéal poétique mallarméen lorsqu'il évoque l'utopie «d'une littérature en transfusion perpétuelle - perfusion transtextuelle $»^{3}$. L'image de la transfusion fait de la transtextualité la matière vitale de l'œuvre littéraire, un de ses éléments définitoires. Par ailleurs l'idée d'une perpétuation indéfinie de la transtextualité, cette représentation d'une littérature qui sans cesse renvoie à ses expressions antérieures et s'en nourrit semble, à partir de l'intertextualité, définir la littérature comme une chaîne infinie, refermée sur elle-même et séparée d'un référent qui n'y aurait de place que sous une forme toujours symbolique, sans cesse recodifiée par la référence à une autre symbolisation littéraire, à un autre univers imaginaire. L'absolu dont rêvait Mallarmé - le Livre - trouverait une expression possible, sous une forme virtuelle et toujours provisoire, dans cette vision de la littérature, envisagée à travers l'intertextualité, comme une totalité « dont tous les auteurs ne font qu'un, et dont tous les livres sont un vaste Livre, un seul Livre infini $»^{4}$.

3 Par son refus du référent, son exigence de la "notion pure", la poétique des symbolistes ne pouvait qu'approfondir et creuser cette pratique intertextuelle conçue comme recodification d'un objet déjà mis en fiction, resymbolisation d'un référent luimême symbolique. En voulant transposer dans le domaine anglais la poésie symboliste française, réécrire Mallarmé et, à travers lui, la totalité de la littérature, Arthur Symons a voulu pousser à son degré ultime cette relation intrinsèque entre poésie et 
intertextualité. Car il a aussi, par ce biais, exploré un autre aspect de l'intertextualité, exposé par Laurent Jenny, celui qui consiste à l'envisager à la fois «comme détournement culturel » et « comme réactivation du sens $»^{5}$.

4 Ainsi fonctionne en particulier son recueil Images of Good and Evil, publié en 1899. L'intertextualité est parfois une référence explicite, comme dans la parenthèse qui suit le titre de «The Two Blind Men» («Les deux aveugles ») ${ }^{6}$ : "From the Neapolitan of Salvatore Di Giacomo ». Le poème se présente alors lui-même comme une adaptation, voire une pure traduction d'un poème écrit en dialecte napolitain. Dans le tableau général des pratiques hypertextuelles qu'il élabore dans Palimpsestes (1982), Gérard Genette range justement la traduction dans la catégorie «transposition ». Il rend ainsi compte d'une fonction de l'hypertextualité : le transfert, l'assimilation par le texte récepteur et son environnement culturel. Par la traduction, Symons transporte en effet dans le domaine anglais une situation culturelle étrangère mais qui, par ses analogies, éclaire la position du poète irlandais de langue anglaise. Di Giacomo, poète et romancier italien né et mort à Naples (1860-1934), compose ses chansons en dialecte napolitain, la langue natale, native et toujours préservée, contrairement au gaëlique perdu. La quête de la langue originelle se fait donc par le truchement de l'intertextualité, par la médiation à une source symbolique où la langue même confère un sens spécifique au modèle.

5 Marc Eigeldinger analyse ce fonctionnement poétique lorsqu'il aborde les « fonctions » de l'intertextualité : «la principale fonction de l'intertextualité est transformatrice et sémantique. Il ne s'agit pas de reproduire à l'état brut le matériau d'emprunt, mais de le métamorphoser et de le transposer $»^{7}$. Le « détournement culturel » dont parle Laurent Jenny permet alors une resémantisation. Car on peut très bien lire, dans les « deux hommes aveugles " que fait dialoguer le poème, la rencontre des deux poètes que permet le jeu de l'intertextualité, l'un aveugle de naissance, l'autre qui l'est devenu. C'est ce dernier qui pose la question: "Who is your Serafina?» Par l'allusion aux Séraphins, bien sûr, c'est au départ, comme dans le poème de Di Giacomo, à la vision déterminant la vocation d'Isaïe que renvoie le poème ${ }^{8}$. Et, selon une image traditionnelle, il oppose à la vision sensible dont sont dépourvus les personnages celle, intérieure ou idéale, de l'homme inspiré ou de l'artiste: "she is beautiful? - Yes, beautiful ». Le poème peut se lire selon un point de vue métaphysique ou esthétique, mais l'intertextualité ajoute à la version de Symons une dimension supplémentaire : par la référence à un objet littéraire, qui se rapporte d'ailleurs lui-même à une source symbolique - la Bible-, le poème peut renvoyer implicitement à une origine mystérieuse de l'art, la langue perdue hors de portée de l'aveugle de naissance. La cécité prend alors sens à l'intérieur d'une gigantesque synesthésie qui définit l'univers des deux aveugles,

The perfume of the garden, and the sun

Shone in the sky, the sun that is of gold.

(Le parfum du jardin et le soleil,

Qui brillait dans le ciel, le soleil qui est d'or).

6 La référence au soleil, récurrente dans le poème, peut également se lire à l'intérieur d'une intertextualité plus vaste, d'un vaste brassage symbolique intégrant la présence du texte biblique. Car seraphim, en hébreu - l'autre langue sacrée et perdue - signifie littéralement «les brûlants ». Cette appréhension globale de l'univers où se mêlent perceptions tactiles et visuelles se rapporte à une conception de la poésie indissociable 
de la langue, langue perdue conçue non seulement comme vecteur d'une identité culturelle mais comme instrument sacré par quoi se dit le mystère du monde.

7 Dans Palimpsestes, Gérard Genette, parce qu'il définissait trop simplement l'intertextualité comme "présence effective d'un texte dans un autre ", en distinguait trois formes : la citation, le plagiat et l'allusion'. On voit à quel point cette typologie, qui en reste aux formes circonscrites, conscientes et clairement délimitées du rapprochement des textes, est loin de rendre compte de l'entreprise poétique de Symons. Le poème, chez lui, se rapporte certes explicitement à un autre, mais pour devenir, à travers ce rapprochement, écho de voix innombrables, parole universelle dont la source justement se perd. Toute entreprise typologique se heurte à la même impasse, à la même incapacité de rendre compte de la vertu poétique de l'intertextualité. Annick Bouillaguet n'y échappe pas non plus lorsqu'elle ajoute aux trois cas évoqués par Gérard Genette une quatrième forme, elle aussi explicite: la «référence $»^{10}$. Par sa forme circonscrite, la notion ne peut rendre compte d'une pratique visant à envisager l'intertextualité comme tremplin destiné à ouvrir le texte vers la totalité de la littérature, à le transformer en caisse de résonance pour l'ensemble de la poésie passée.

8 Par ailleurs la poétique de Symons fait apparaître, dans " The Two Blind Men ", l'autre aspect de l'intertextualité. Si elle vise à mettre à distance le référent pour définir l'acte poétique comme resymbolisation ou resémantisation d'un objet symbolique, elle ne construit pas cependant le texte poétique comme système clos. La mise à distance du monde, liée à une quête de vérités plus essentielles, conduit à l'infini de l'art vers lequel s'ouvre chaque texte. C'est en ce sens que Michael Worton et Judith Still soulignent que le texte, à travers son réseau intertextuel, « ne peut exister comme un tout hermétique et auto-suffisant, et par là ne fonctionne pas comme un système clos $»^{11}$. De fait, le texte, chez Symons, opère une irradiation. Le poème prend place et se lit à l'intérieur du vaste tissage formé par la littérature, métaphore employée à juste titre par bon nombre de théoriciens de l'intertextualité. Nathalie Limat-Letellier et Marie MignetOllagnier rappellent que l'étymologie du radical comme du prédicat « évoque la qualité du texte comme "tissage", "trame" » ce qui implique d'aborder la littérature à travers "l'idée de réseau, d'intersection $~^{12}$. Le texte prend sens par son rapport à ceux qu'il évoque et dont il se nourrit, si bien que l'intertextualité, plus que d'un emprunt, prend la forme d'une "trace », selon l'expression de Michaël Riffaterre. C'est effectivement ainsi que fonctionnent, dans le poème de Symons, le texte de la Bible, la langue hébraïque. Marc Eigeldinger, qui rappelle cependant que l'intertextualité « ne saurait se confondre avec l'établissement des sources", préfère la notion de "greffe " ${ }^{13}$. Nathalie Limat-Letellier recourt à la même formule et la justifie en examinant le fonctionnement de l'intertextualité comme «l'engendrement d'un nouveau texte par dérivation $»^{14}$. La reprise du poème de Di Giacomo, dans un nouveau contexte et dans une langue différente, opère ce processus de dérivation en donnant naissance à un poème différent, chargé d'une signification différente à partir des mêmes symboles.

Il peut alors être utile de retourner à l'origine de la notion d'intertextualité, introduite dans la critique française par Julia Kristeva autour de $1966^{15}$. Dans son article « Le mot, le dialogue et le roman", celle-ci distingue deux axes: l'axe horizontal, formé par le sujet et le destinataire, et l'axe vertical, constitué par le texte et le contexte. Elle conclut de ce schéma que «le mot (le texte) est un croisement de mots (de textes) où on lit au moins un autre mot (texte) $»^{16}$. La transplantation d'un mot ou texte dans un 
autre modifie les deux axes, en transformant au moins deux paramètres, le destinataire et le contexte. Si bien que le même texte représentera, à l'intérieur de l'hypotexte et de l'hypertexte $^{17}$, deux constructions symboliques totalement différentes. En d'autres termes, on ne peut se contenter de lire le poème de Symons comme celui de Di Giacomo, comme s'il s'agissait d'une simple traduction en une autre langue (cette notion étant du reste elle-même suspecte). Le jeu de l'intertextualité a conduit à créer un nouveau poème à la signification différente: celle du poème de Di Giacomo est contenue, réassimilée et transformée, à la manière d'un greffon, dans le poème de Symons.

10 Les deux axes de Julia Kristeva conduisent à prendre en compte, pour la définition du fonctionnement de l'intertextualité, un extérieur du texte littéraire: sa lecture, sa réception. C'est aussi en fonction de ce que H.-R. Jauss appelle un " horizon d'attente " que le texte qui en contient un autre prend un sens nouveau. Michaël Riffaterre place même le lecteur au centre de sa définition de l'intertextualité en tant que critère de littérarité : "L'intertextualité est la perception, par le lecteur, de rapports entre une œuvre et d'autres $[. .]$.$» . Or pour lui, c'est dans « la perception de ces rapports »^{18}$, non dans leur existence, que réside la littérarité.

11 La pratique poétique de l'intertextualité doit donc consister essentiellement à jouer avec un acquis supposé du lecteur, plus précisément à prendre à contre-pied un lien déjà établi, dans l'esprit du lecteur, entre une image et un sens, afin de produire de nouvelles significations par le jeu de la réécriture. La source du jeu intertextuel peut donc prendre une forme plus diffuse, celle de ce que Marc Eigeldinger appelle « un langage culturel »: « Toute insertion d'un langage culturel dans le texte littéraire peut devenir objet d'intertextualité $»^{19}$. Et il distingue cinq principaux champs intertextuels : le champ de la littérature, le champ artistique, le champ mythique, le champ biblique et celui de la philosophie. A côté du champ de la littérature et du champ biblique, Symons a abondamment exploré le champ mythique et plusieurs poèmes permettent de montrer comment l'intertextualité se déploie à travers le mythe pour construire un jeu complexe de symbolisation.

12 L'exemple du poème "The Chimaera ${ }^{20}$ est à cet égard révélateur de la complexité des références mises en œuvre par Symons. Symons y combine deux univers imaginaires habituellement séparés l'un de l'autre dans l'esprit du lecteur : celui de la mythologie antique et celui d'une autre mythologie, celle des Minnelieder, eux-mêmes combinés à l'univers chrétien. Le glissement entre ces univers s'opère par le système des images. Ainsi la Chimère, caractérisée d'abord, à travers une métaphore, comme "wandering angel» («ange voyageur»), ouvre-t-elle ensuite l'univers mythologique par le biais d'une comparaison. C'est la chimère qui prend la parole et compare le narrateur à Psyché («Like Psyche from his naked face »). La référence chrétienne semble encadrer le poème et lui donner, à travers l'imagerie mythologique, son véritable sens. Le narrateur évoque l'objet de sa quête, « The most fair gate of Hell » (« la plus belle porte de l'Enfer »). L'image d'un "white angel » est reprise vers la fin, et les derniers vers évoquent, à travers Dieu, une éternité dont le sens ne peut être que chrétien.

Les figures au centre de ce poème placent au premier plan de ces univers imaginaires des jeux intertextuels précis ou diffus. L'entrelacs des références peut lui-même être lu comme une référence à La Tentation de saint Antoine de Flaubert (1874). Dans la section "monstres» de son "Bestiaire mythologique», André Siganos souligne ce rapprochement entre références chrétiennes et mythologiques, à travers l'image du 
monstre, être composite, fondamentalement hétérogène : «La Chimère et le Sphinx avaient auparavant tenté un abominable coït devant le saint éperdu » ${ }^{21}$. Et Jeanne Bem note comment, chez Flaubert déjà, l'entrecroisement des sources vise à bâtir un univers nouveau, comment le texte se tissait par la convergence d'échos littéraires : « en faisant dialoguer le Sphinx et la Chimère, Flaubert croise deux séries mythiques traditionnelles et fournit le support textuel d'un mythe nouveau $»^{22}$. Symons ne fait pas dialoguer la Chimère avec le Sphinx. Mais en croisant lui aussi les univers autour de la Chimère, il rappelle le texte de Flaubert et, dans le dialogue du narrateur avec la Chimère, suggère implicitement, par le biais d'une intertextualité subtile, une identification possible du narrateur avec le Sphinx. Le jeu intertextuel inscrit plus généralement le poème de Symons dans un univers décadent, puisqu'il se redouble d'une référence sous-jacente au roman de Huysmans, A Rebours, où le dialogue du Sphinx et de la Chimère exalte Des Esseintes. Ce second niveau d'intertextualité est souligné par la forme du dialogue. Jeanne Bem note en effet qu' en passant dans le texte de Huysmans, le texte de Flaubert est curieusement rethéâtralisé, puisque les répliques qu'échangent les deux monstres sont récitées par une ventriloque, un monstre aussi à sa façon ${ }^{23}$. En renvoyant implicitement au texte de Huysmans, Symons affecte à l'intertextualité un second niveau de sens : derrière le dialogue entre l'Inconnu ou le Secret et la Fantaisie, suggéré dans son propre poème, derrière même la conception du texte comme tissage d'un matériau déjà poétisé, significations qui se dégagent de la référence indirecte à Flaubert, il fait de l'intertextualité, en la dédoublant, la fin ultime de la poésie, en tant qu'elle a pour fonction de reconstruire, de repoétiser le symbole. Le texte poétique se définit alors lui-même comme symbole, sens énigmatique, idée qui se dégage de l'image du Secret, significativement absente du poème : le Sphinx n'y est présent que par le jeu de l'intertextualité qui devient alors dépositaire du sens ultime du poème. De même que Mallarmé célébrait «l'absente de tous bouquets $»^{24}$, Symons place dans l'absence, dans une littérature autre, présente ailleurs, et de ce fait absolue, la signification de son texte. Enfin le monstre, figure sous-jacente de ce système de références, devient une image ou une projection du texte lui-même, qui se nourrit et se construit en combinant et en mêlant des sources hétérogènes.

Dans le poème, l'explicite vient contredire néanmoins la référence implicite au Sphinx et lui superposer un autre niveau de sens à travers un autre jeu intertextuel. Si le narrateur, par le jeu intertextuel, peut être rapproché dans une certaine mesure du sphinx, il est explicitement comparé à Psyché. Or la comparaison fait surgir un autre texte à la conscience du lecteur, la fable d'Amour et de Psyché des Métamorphoses d'Apulée. Et le jeu des images reconstruit la trame d'Apulée. Chez Apulée, Vénus avait demandé à Cupidon, par le mal d'amour, de châtier Psyché. Plus tard, elle envoie Psyché aux enfers pour y chercher un coffret, ce qui la perd d'abord. Le poème, de même qu'il rassemble des sources hétérogènes, réconcilie les deux figures: le narrateur, comparé à Psyché, est "Born under Venus " (" né sous Vénus »). Par cette double référence aux sens et à l'âme, Symons exprime l'aspiration à une réconciliation des deux dimensions de l'homme. On pourrait se perdre à chercher des références intertextuelles précises dans le poème de Symons. Du conte de La Fontaine (1669) à la tragédie-ballet de Corneille, Molière et Philippe Quinault (1771), nombreuses ont été les illustrations littéraires qui ont exalté la sensualité de la figure de Psyché. Mais le texte de Symons présente une allusion plus concrète à La Généalogie des dieux (1347-59), où Boccace voit, dans la figure de Psyché, l'allégorie de l'âme succombant, par orgueil et par désobéissance, aux tentations de la vie végétative et de la vie sensitive, mais 
finissant par revenir à Dieu. Le narrateur du poème de Symons, tenté par la porte des Enfers, s'exclame néanmoins :

[...] Some day, Chimaera, I shall find the way!

Un jour, Chimère, je trouverai le chemin!

Et la fin du poème se construit comme un retour vers Dieu, dans l'éternité de la mort. C'est donc encore le jeu intertextuel qui éclaire le mélange des sources, le sens chrétien d'une imagerie païenne.

Un troisième univers imaginaire s'ajoute au poème à travers la référence à Tannhäuser :

Chimaera, I have been among

The loving people, who yet throng

The twilight about Tannhäuser [...]

Chimère, j'ai été parmi

Le peuple aimant, qui déferle encore

Sur le crépuscule, autour de Tannhäuser.

17 Bien sûr, la référence à Tannhäuser introduit un autre univers, celui de l'Allemagne médiévale. Mais elle fait sens, également, dans le traitement symbolique du référent. Car Tannhäuser est la seule figure historique du poème. Le "peuple aimant» rapproche alors les figures mythologiques, purs symboles, d'un univers réel, celui des croisades. Ou plutôt, il procède à une symbolisation du référent historique. Celui-ci est ainsi indissociable d'une mise en fiction, elle-même due en partie au fait que Tannhäuser, figure du Minnesang à son déclin, est également une image du Poète. Par ailleurs si le personnage historique s'est probablement illustré au cours de la croisade de 1228 , il devient, dès 1451 , héros de légende dans le poème de Hermann von Sachsenheim. Dans leur quête de figures fondatrices de la littérature nationale, les frères Grimm dans leurs Deutsche Sagen, tout comme Heine dans ses Elementargeister, font allusion à Tannhäuser.

18 Mais au moment où Symons écrit son poème, la référence la plus prégnante est déjà l'opéra de Wagner (1845). Car le point de départ de Wagner est lui-même une double référence intertextuelle. Wagner connaissait les Sagen (1836) de L. Bechstein et l'essai de C.S. Lucas Über den Wartburgkrieg (1838), textes qui suggéraient une autre hybridation en identifiant Tannhäuser à Heinrich von Oefterdingen. Par le jeu intertextuel, Symons désigne ainsi l'intertextualité comme fondement de son entreprise de reconstruction symbolique.

On voit dans quelle mesure, sous sa forme diffuse, la pratique de l'intertextualité chez Symons correspond aux fonctions de l'allusion telle que l'a envisagée Ruth Amossy. Par sa nature, celle-ci « exhibe en effet le renvoi à un discours antérieur dont elle fait jouer les éléments dans une texture nouvelle $»^{25}$. La texture nouvelle permet de produire un nouveau niveau de symbolisation, tout en conservant un écho de la signification du texte-source. Mais chez Symons, ce sens supplémentaire est à lire dans l'intertextualité elle-même, qui devient presque la finalité du poème. L'irradiation du poème à travers le champ du littéraire le déborde finalement : dans le poème, la référence à Tannhäuser évoque un tableau, mais l'intertextualité suggère l'opéra. La symbolisation sort du domaine de l'intertextualité pour englober la totalité de l'art. Dans son essai consacré à Dante Gabriel Rossetti, il montre qu'il est impossible de parler de ses tableaux sans penser à ses poèmes ${ }^{26}$ et souligne, à travers l'exemple de la Salomé de Moreau reconstruite par Huysmans dans A Rebours, que la peinture déborde dans la littérature : 
« and Moreau will live in the pages which Huysmans has written about him at least as long as in his pictures» ("et Moreau vivra dans les pages que Huysmans lui a consacrées au moins aussi longtemps que dans ses tableaux $»)^{27}$.

Par le brassage des textes, des genres, des arts, des univers imaginaires, Symons recherche l'unité de l'art et de toute construction symbolique. L'intertextualité se prolonge dans ce que l'on pourrait appeler une intergénéricité, et celle-ci a le même sens. Toutes deux visent à mettre à distance le référent, à l'abolir en quelque sorte derrière l'écran formé par un objet artistique. Cette recherche, à travers l'intertextualité, d'une unité fondamentale - et peut-être originelle - de la littérature, et, par delà l'intertextualité, la recherche de l'unité de toute construction symbolique, vise à faire du symbole - non de ce qu'il dit - la fin ultime de l'art. Le symbole vise à se dire lui-même dans une poésie réflexive qui s'étend aux autres arts, mais pour mieux affirmer la suprématie du langage comme objet symbolique.

21 L'entreprise de Symons explore donc les confins de l'intertextualité. Elle définit la poésie comme œuvre de langage en visant, par le jeu intertextuel, à abolir toute référentialité. De fait, la poétique de Symons illustre bien cette assertion de Michaël Riffaterre, pour qui « dans l'œuvre littéraire, les mots ne signifient pas par référence à des choses ou à des concepts [...], mais par référence à ces complexes de représentations déjà entièrement intégrés à l'univers langagier ${ }^{28}$. L'intertextualité rappelle plus largement que le sens de la poésie ne réside pas dans le rapport des mots aux choses, mais dans le rapport des mots à un univers imaginaire lui-même littéraire, c'est-à-dire langagier. Elle pose, en définitive, la réflexivité du langage poétique.

\section{NOTES}

1. «Crise de vers », in : Euvres complètes,. Ed. par Henri Mondor et G. Jean-Aubry, Paris Gallimard («Bibliothèque de La Pléiade »), p. 367.

2. Ibid., p. 368.

3. Palimpsestes, la littérature au second degré. Paris, Seuil (coll. « Poétique »), 1982, p. 453.

4. Idem.

5. «La Stratégie de la forme », Poétique, 1976, n² 27, p. 279.

6. Images of Good and Evil (1899). New York, Wodstock Books, 1996, p. 66-67.

7. Mythologie et Intertextualité. Genève, Slatkine, 1987, p. 16-17.

8. Is. $6: 2-3$.

9. Op. cit., p. 8.

10. « Une typologie de l'emprunt », Poétique, n 80, nov. 1989, p. 496.

11. Michael Worton et Judith Still (ed.), Intertextuality. Theories and Practices. Manchester, Manchester University Press, 1990, p. $1:$ « The theory of intertextuality insists that a text [...] cannot exist as a hermetic or self-sufficient whole, and so does not function as a closed system.» 
12. Nathalie Limat-Letellier et Marie Mignet-Ollagnier (ed.), L'Intertextualité. Paris, Les Belles Lettres (Annales littéraires de l'Université de Franche-Comté), 1998, "Préface », p. 17.

13. Marc Eigeldinger, op. cit., p. 9.

14. « Historique du concept d'intertextualité », in : op. cit., p. 7.

15. Dans deux articles repris dans Semiotikè. Recherches pour une sémanalyse. Paris, Seuil (coll. « Tel Quel »), 1969 : « Le mot, le dialogue et le roman », daté de 1966 et « Le texte clos », daté de 1966-67.

16. Semiotikè, op. cit., p. 145-6.

17. Voir Gérard Genette, Palimpsestes, op. cit., p. 11-12.

18. « La trace de l'intertexte », La Pensée, $\mathrm{n}^{\circ} 215$, oct. 1980, p. 4.

19. Op. cit., p. 15.

20. Op. cit., p. 49-56.

21. "Bestiaire mythologique », in : Pierre Brunel (ed.), Dictionnaire des mythes littéraires. Nouvelle édition augmentée. Monaco, éditions du Rocher, 1988, p. 228.

22. « La Tentation de saint Antoine », in : Pierre Brunel, op. cit., p. 1350.

23. Ibid., p. 1351.

24. "Crise de vers ", in : CEuvres complètes, op. cit., p. 368.

25. Les Jeux de l'allusion littéraire dans "Un Beau Ténébreux" de Julien Gracq. Neuchâtel, La Baconnière, 1980, p. 20.

26. Dante Gabriel Rossetti. London, Fisher-Unwin, 1910, p. 5 : « It is impossible to speak of his pictures without thinking of his poems ».

27. Ibid., p. 9.

28. « L'Intertexte inconnu », Littérature, n 41, 1981.

\section{RÉSUMÉS}

L'étude envisage les aspects, significations et enjeux poétiques de l'intertextualité chez Symons : allusions ou références aux textes antiques, traductions ou réécritures (parfois allusives), retraitement de sujets mythologiques avec des références précises à des traitements antérieurs. Dans tous les cas, ces jeux d'intertextualité visent à mettre à distance le référent, à l'abolir en quelque sorte derrière l'écran formé par un objet littéraire. Cette intertextualité irradiante s'érige en pratique poétique par excellence dans la mesure où l'objet resymbolisé est en définitive la poésie elle-même.

\section{AUTEURS}

\section{MICÉALA SYMINGTON}

Université de Nice-Sophia Antipolis

BERNARD FRANCO

Université de Paris-Sorbonne (Paris IV) 